\title{
Interplay of Floquet Lifshitz transitions and topological transitions in bilayer Dirac materials
}

\author{
Priyanka Mohan ${ }^{1,2}$ and Sumathi Rao ${ }^{2}$ \\ ${ }^{1}$ Department of Theoretical Physics, Tata Institute of Fundamental Research, Homi Bhabha Road, Mumbai 400005, India \\ ${ }^{2}$ Harish-Chandra Research Institute, HBNI, Chhatnag Road, Jhunsi, Allahabad 211 019, India.
}

\begin{abstract}
We show how transitions between different Lifshitz phases in bilayer Dirac materials with and without spin-orbit coupling can be studied by driving the system. The periodic driving is induced by a laser and the resultant phase diagram is studied in the high frequency limit using the BrillouinWigner perturbation approach to leading order. The examples of such materials include bilayer graphene and spin-orbit coupled materials such as bilayer silicene. The phase diagrams of the effective static models are analyzed to understand the interplay of topological phase transitions, with changes in the Chern number and topological Lifshitz transitions, with the ensuing changes in the Fermi surface. Both the topological transitions and the Lifshitz transitions are tuned by the amplitude of the drive.
\end{abstract}

\section{INTRODUCTION}

Bilayer graphene $e^{1-8}$ has been intensely studied in the last few years, mainly due to the tunability of its bandgap by an electric field. The two layers can be oriented differently, but what is most commonly studied is the $\mathrm{AB}$ or Bernal stacking, which is when the top layer is shifted so that half its atoms lie directly over the center of the hexagon of the lower graphene sheet. The band structure no longer has Dirac cones and the charge carriers in bilayer graphene are expected to be massive Dirac fermions. On the other hand, for spin-orbit coupled materials like silicene (and other materials like germanene and stanene $)^{9} \sqrt{13}$, which have a buckled structure, tunability is already present in a monolayer, and it is well-known that the band structure can be tuned between band insulators and topological insulators through a metallic phase by applying an electric field. However, bilayer silicene ${ }^{14}[18$ (and other spin-orbit coupled materials) have even richer physical properties due to the interplay of buckling and stacking, and are beginning to be studied in detail.

Bilayer silicene is a trivial topological insulator, unlike monolayer silicene, due to the lack of topologically protected edge states, in the presence of interlayer spinorbit coupling and Rashba spin-orbit coupling. However, it has been argued that many of its physical properties are similar to those of topological insulator and it should be considered a quasi-topological insulator ${ }^{14}$. At a critical electric field, the quasi-topological phase of bilayer silicene makes a transition to a band insulator, but due to trigonal warping, there are several such critical electric fields where the band closes, and the band structure is thus controllable by the electric field to an even larger extent than in bilayer graphene. The electronic structure and properties of bilayer silicene are also strongly stacking dependent $t^{15 / 16}$ and recent work has also investigated the effect of perpendicular electric and magnetic fields on bilayer silicene.

The study of light-matter interaction has become increasingly popular in the last few years and new devel- opments in laser studies have led to the possibility of Floquet engineering 1920 which is the generation of new Hamiltonians that do not exist in static systems. In particular, besides experiments in cold atom ${ }^{211}$ and photonic systems $^{22}$, with the emergence of new topological phases in condensed matter systems ${ }^{23}$, the field of Floquet topological insulators ${ }^{24}$ has shot into prominence in recent times.

The possibility of tuning the band-gap in graphene and bilayer graphene using light greatly increases potential applications. It has also been shown $\frac{38139}{\text { that bilayer }}$ graphene is an ideal system to study Lifshitz transitions, because the parabolic dispersion in Bernal stacked bilayer graphene is not protected by the crystal symmetry and is trigonally deformed at the lowest energies due to next nearest neighbour interlayer hopping, giving rise to four Dirac cones. Under an inter-layer voltage bias, the Dirac points can move and cause a Lifshitz transition - i.e., can change the topology of the Fermi surface. In this paper, we study the effect of shining light on bilayer graphene as well as bilayer silicene and other spin-orbit coupled Dirac materials, in the high frequency limit using the BrillouinWigner perturbation approach. It was shown recently ${ }^{39}$ that three van Hove saddles merge at a multi-critical Lifshitz point in bilayer graphene and it was shown that four different phases with different Fermi surface topologies occur. One of the main results that we obtain in this paper is the effect of driving on the phase diagram with these four different Fermi surface topologies. The driving also induces new topological phases with different Chern numbers and we obtain those phases as well. Although the effect of irradiation on bilayer graphene ${ }^{40 \mid 41}$ has been recently studied, here we focus specifically on the the Lifshitz transitions and how they can be affected by driving. Moreover, we include effects of buckling as well as spin-orbit coupling so that other materials such as silicene, germanene, etc can also be incorporated, where far fewer studies exist.

The plan of our paper is as follows. In Sec. II, we briefly review the Lifshitz transition in bilayer graphene to set the notation. In Sec. III, we include the intrin- 
sic spin-orbit coupling and the buckling, which are the hallmarks of silicene and other spin-orbit coupled materials and emphasize how the Lifshitz transition in these materials differs from graphene. In Sec. IV, we include periodic driving and study the Brillouin-Wigner (B-W) perturbation expansion, without and with spin-orbit coupling, in bilayer systems, generalizing earlier results $35 \mid 36$ on single layers ands obtain the effective static Hamiltonian. In Sec V, we obtain the phase diagrams and show how the Lifshitz transition gets modified in the presence of light for both bilayer graphene and bilayer spin-orbit coupled materials. We end with a small discussion in Sec. VI, where we briefly discuss how the Lifshitz transitions may be observed in a real system.

\section{BILAYER GRAPHENE AND THE LIFSHITZ TRANSITION}

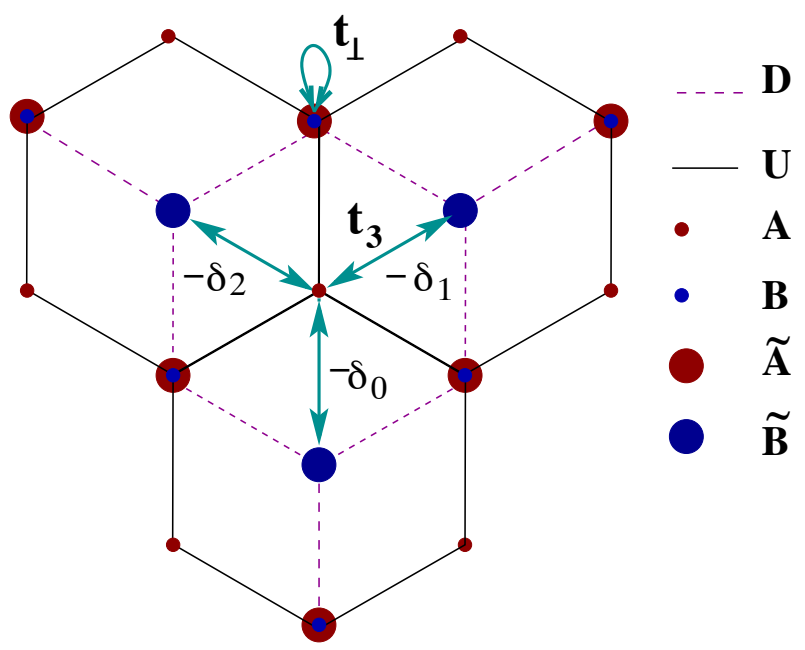

FIG. 1: Bilayer model viewed from above. The small dots (red and blue) denote the carbon atoms (on A and B sub-lattices) in one layer (U or up) whereas the big dots denote the same in the other layer ( $\mathrm{D}$ or down).

The Hamiltonian of bilayer graphene can be written as the sum of the Hamiltonians of the individual layers and the inter-layer couplings between them. There are several different possible stackings including layers which are twisted with respect to one another. However, here we shall work with the most common Bernal or AB stacking. Although some details of the phase diagram may change in the other cases, we expect that the general features of the phase diagram will essentially remain the same. The Hamiltonian for Bernal stacking is given by

$$
H_{B L}=H_{S L}^{U}+H_{S L}^{D}+H_{\text {inter }}
$$

where the $H_{S L}^{U}, H_{S L}^{D}$ are the two single layer Hamiltonians for the up and down layers given by

$$
H_{\mathrm{SLG}}=-t \sum_{\langle i j\rangle, \sigma} a_{i, \sigma}^{\dagger} b_{j, \sigma}+h . c
$$

and $H_{\text {inter }}$ is the interlayer coupling. The index $\sigma$ refers to real spin and is usually dropped in the graphene context, since the Hamiltonian can be written independently for each spin. The sub-lattices of the two layers are denoted as $A, B$ for the up layer and $\tilde{A}, \tilde{B}$ for the down layer and the the Hamiltonian for the inter-layer hopping $H_{\text {inter }}$ is given by,

$$
\begin{aligned}
H_{\text {inter }} & =t_{\perp} \sum_{i \in \tilde{A}, j \in B}\left(\tilde{a}_{i \sigma}^{\dagger} b_{j \sigma}+b_{j \sigma}^{\dagger} \tilde{a}_{i \sigma}\right) \\
& +t_{3} \sum_{i \in A, j \in \tilde{B}}\left(a_{i \sigma}^{\dagger} \tilde{b}_{j \sigma}+\tilde{b}_{j \sigma}^{\dagger} a_{i \sigma}\right) .
\end{aligned}
$$

As shown in Fig,1, there are two distinct types of hopping terms between the layers. The hopping term $t_{\perp}$ is between sub-lattices $B$ and $\tilde{A}$ which are separated by $2 L$ in the direction perpendicular to the planes, whereas the interlayer hopping term $t_{3}$ is between sub-lattices $A$ and $\tilde{B}$ separated by $2 L$ in the perpendicular direction and also one bond length along the planes. The magnitude of both interlayer hopping terms are $t_{3}, t_{\perp} \sim 0.1 t$, where $t_{\perp} \gtrsim t_{3}$. Here, we have not considered the interlayer spin-orbit coupling.

The total low-energy Hamiltonian in the vicinity of the Dirac points (for each spin) can be obtained in the momentum space and is given by

$$
\psi_{q}^{\dagger} H_{\eta} \psi_{q}=\psi_{q}^{\dagger}\left(\begin{array}{cccc}
L E_{z} & \frac{3 a t}{2}\left(\eta q_{x}-i q_{y}\right) & 0 & -\frac{3 a t_{3}}{2}\left(\eta q_{x}+i q_{y}\right) \\
\frac{3 a t}{2}\left(\eta q_{x}+i q_{y}\right) & L E_{z} & t_{\perp} & 0 \\
0 & t_{\perp} & -L E_{z} & \frac{3 a t}{2}\left(\eta q_{x}-i q_{y}\right) \\
-\frac{3 a t_{3}}{2}\left(\eta q_{x}-i q_{y}\right) & 0 & \frac{3 a t}{2}\left(\eta q_{x}+i q_{y}\right) & -L E_{z}
\end{array}\right) \psi_{q}
$$

where $\psi_{q}=\left(\begin{array}{llll}a_{q} & b_{q} & \tilde{a}_{q} & \tilde{b}_{q}\end{array}\right)^{T}, \eta= \pm 1$ for $K, K^{\prime}$ is the pseudo-spin index and $a$ is the nearest-neighbour dis- tance. An electric field added perpendicular to the layers will add a potential difference between the two layers. The four eigenvalues of the above Hamiltonian are given by 


$$
\begin{aligned}
\epsilon_{q, \eta} & = \pm \frac{1}{\sqrt{2}}\left(2 L^{2} E_{z}^{2}+\frac{9 a^{2} q^{2}}{4}\left(2 t^{2}+t_{3}^{2}\right)+t_{\perp}^{2}\right. \\
& \left. \pm\left(t_{\perp}^{4}+9 a^{2} q^{2} t^{2}\left(4 L^{2} E_{z}^{2}+t_{\perp}^{2}\right)+\frac{9 a^{2} q^{2} t_{3}^{2}}{4}\left(9 a^{2} q^{2}\left(t^{2}+\frac{t_{3}^{2}}{4}\right)-2 t_{\perp}^{2}\right)-27 \eta a^{3} t^{2} t_{3} t_{\perp} q_{x}\left(q_{x}^{2}-3 q_{y}^{2}\right)\right)^{1 / 2}\right)^{1 / 2}
\end{aligned}
$$

where $q^{2}=q_{x}^{2}+q_{y}^{2}$. Precisely at the $K$ and $K^{\prime}$ points, the four eigenvalues reduce to

$$
\epsilon_{\eta}= \pm L E_{z}, \pm \sqrt{L^{2} E_{z}^{2}+t_{\perp}^{2}}
$$

The linear dispersion of the two graphene layers become quadratic on the addition of the coupling $t_{\perp}$. Switching on the $t_{3}$ term adds trigonal warping to the bands near the $K\left(K^{\prime}\right)$ points. This results in the quadratic band dividing into four Dirac cones: one at the $K\left(K^{\prime}\right)$ point and three satellite cones around it. The three satellite Dirac points are situated at $\left(\frac{\eta t_{\perp} t_{3}}{3 a t^{2}}, \pm \frac{t_{\perp} t_{3}}{\sqrt{3} a t^{2}}\right)$ and $\left(-\frac{2 t_{3} t_{\perp}}{3 a \eta t^{2}}, 0\right)$. These points can be easily computed by checking for nodes at non-zero momenta along the $q_{y}=0$ axis for $E_{z}=0$ and using the trigonal symmetry. Each of the satellite Dirac points is separated from the $K\left(K^{\prime}\right)$ point by a van-Hove singularity, which here is a maximum. At the satellite Dirac points, Eq5 takes the form,

$$
\begin{aligned}
\epsilon_{\text {satellite }} & = \pm\left(2 L^{2} E_{z}^{2} t^{4}+\left(t^{2}+t_{3}^{2}\right)^{2} t_{\perp}^{2}\right. \\
& \left. \pm t_{\perp}\left(16 L^{2} E_{z}^{2} t^{6} t_{3}^{2}+\left(t^{2}+t_{3}^{2}\right)^{4} t_{\perp}^{2}\right)^{1 / 2}\right)^{1 / 2}
\end{aligned}
$$

Note that two of the values in both Eq,6]and Eq77are zero when $E_{z}=0$. As we increase the value of $E_{z}$, the system becomes gapped and the van-Hove singularity moves towards the $K\left(K^{\prime}\right)$ points. The four Dirac cones are replaced by electron-like pockets here. At a critical $E_{z}$ given by $L E_{z}^{\text {critical }}=t_{\perp} t_{3} / 2 t$, the van-Hove singularities merge causing a Lifshitz multi-critical point 39 . For $E_{z}>E_{z}^{\text {critical }}$, the central electron-like pocket becomes a hole-like one while the other three remain electron-like. In Fig 2, we show the phase diagram with phases with different Fermi surface topologies, similar to the one shown in Ref 39. However, we choose to show it as a function of the external perpendicular electric field $E_{z}$ and the Fermi energy.

To see the different Fermi surface topologies with an increasing electric field we have to include the chemical potential $\mu\left(\varepsilon_{F}\right)$ term in Eq 5 where it has been set to zero. The wavy striped region in Fig 2 is when the Fermi energy or chemical potential lies in the gap between the conduction and valence bands. An increase in the electric field pushes the valence and conduction bands away from each other increasing the striped region. For $E_{z}=0$, shifting the Fermi level upwards will result in four disconnected Fermi surfaces, one from the Dirac cone and one from each of the three satellite cones. This phase forms the red region in the phase diagram. As we increase the external electric field from zero, the distance between the valence and conduction bands at

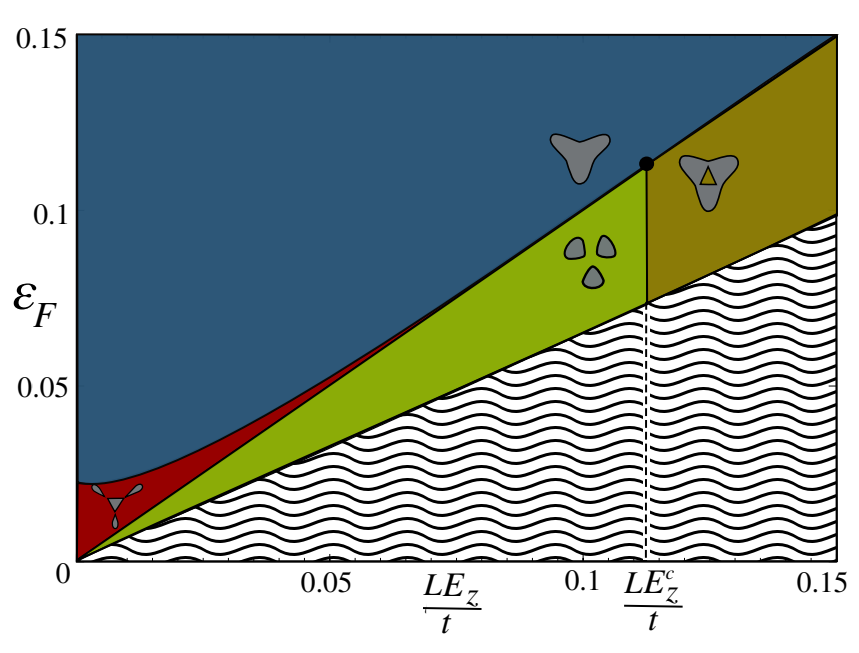

FIG. 2: The Lifshitz phase diagram of bilayer graphene. The different phases are plotted with an increasing electric field. The shape of Fermi surface in each phase is shown in grey color. The black dot at $E_{z}^{c}$ is the Lifshitz multi critical point. The values of inter-layer couplings are $t_{\perp}=0.5 t$ and $t_{3}=0.45 t$ in this phase diagram for illustrative purposes.

$K / K^{\prime}$ points increases further than at the satellite points. When the Fermi level is between the Dirac cone and the satellite cones, the Fermi surface consists of three disconnected regions. This area of the phase diagram is shown in green. The height of the line (measured from zero) separating the blue and red regions in the phase diagram is the height of the van-Hove singularity measured from the point half-way between the valence and conduction bands. Above this phase boundary, the Fermi energy has increased to the level that the Dirac cone and the satellite cones merge to a single band with the topology of a single region. As we increase the external electric field, the three van-Hove singularities move closer to the $K / K^{\prime}$ point and merge at a critical electric field $E_{z}^{c}$. A multicritical Lifshitz point occurs in the phase diagram at the $E_{z}^{c}$ where all the different Lifshitz phases meet. A further increase in electric field causes the electron-like pocket at the $K / K^{\prime}$ point to become hole-like. This phase is the mustard region with the topology of an annulus. The Lifshitz phase transition between the blue and the red phases in the phase diagram is of the 'neck-narrowing type'. As the name indicates, here the Fermi surface get a new topology by pinching off a region. The transition between the green and mustard region is also of the same type. The phase transitions between the red and green 


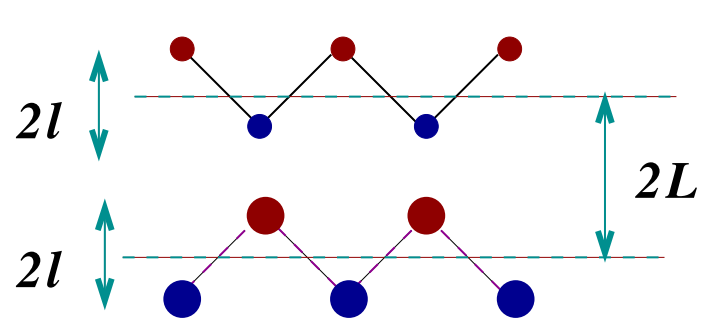

FIG. 3: The different sub-lattices in bilayer silicene viewed horizontally layer by layer. The small and big dots denote the carbon toms in the upper (U) and lower

(D) layers respectively.

phases and the one between the blue and mustard phases is of the 'pocket vanishing type'. Here the topology of the Fermi surface changes when an electron-like pocket becomes hole-like or vice versa.

\section{EXTENSION TO SPIN-ORBIT COUPLED BILAYER MATERIALS}

The difference between graphene and other twodimensional (2D) materials like silicene or germanene is essentially due to the larger size of their atoms which causes buckling of the lattice as well as leading to the presence of an intrinsic spin-orbit coupling. In this paper, we study a general material with arbitrary values for spin-orbit coupling and buckling distance, so that the results can be applied to any of these materials. In the rest of the work, the term silicene is used to represent such a general material.

For a single layer, the Hamiltonian of these materials has additional terms beyond the terms in Eq2 given by,

$$
\begin{aligned}
H_{\text {SLS-extra }} & =\frac{i \lambda \sigma}{3 \sqrt{3}} \sum_{\langle\langle i j\rangle\rangle, \sigma} \nu_{i j}\left(a_{i, \sigma}^{\dagger} a_{j, \sigma}+b_{i, \sigma}^{\dagger} b_{j, \sigma}\right) \\
& +\sum_{i, \sigma} l E_{z}\left(a_{i, \sigma}^{\dagger} a_{i, \sigma}-b_{i, \sigma}^{\dagger} b_{i, \sigma}\right) .
\end{aligned}
$$

Here, $\lambda$ is the spin-orbit coupling between the sites of the same sub-lattice and $2 l$ is the buckling distance as shown in Fig.3. The spin index $\sigma= \pm 1$ corresponding to $\uparrow / \downarrow$ and $\nu_{i j}= \pm 1$ depending on whether the path taken from $j$ to $i$ is clockwise/counter-clockwise. An external electric field $E_{z}$ is added perpendicular to the layers which creates a potential difference between the $A$ and $B$ sublattices and also between the layers.
The Hamiltonian of bilayer silicene has earlier been studied in Ref 14 and its band structure and edge modes were obtained. Here, we review this for two reasons - first, to understand Lifshitz transitions in this model which have not been studied earlier and second, to set our notation for the coupling to photons in the next section. We have two copies of the single layer Hamiltonian given by

$$
H_{S L S}=H_{S L G}+H_{S L S-e x t r a}
$$

and the inter-layer coupling as given in Eq3. Like the case of bilayer graphene, there are two hopping terms between the layers which are separated by a distance $2 L$ (see Fig 3). The hopping term $t_{\perp}$ is between sub-lattices $B$ and $A$ which are separated by $2(L-l)$ in the direction perpendicular to the planes. The other interlayer hopping term $t_{3}$ is between sub-lattices $A$ and $\tilde{B}$ separated by $2(L+l)$ in the perpendicular direction. Due to the individual buckling of both layers, all the four sublattices in bilayer silicene are on four different planes and therefore at four different potentials when $E_{z}$ is applied. The magnitude of both interlayer hopping terms $t_{3}, t_{\perp}$ are $\sim 0.1 t$, and furthermore, $t_{\perp} \gtrsim t_{3}$. Note that the interlayer spin-orbit coupling has been set to zero, so the model still has spin conservation. However, this conservation is not protected by a symmetry and so bilayer silicene is a 'quasi-topological insulator 14 .

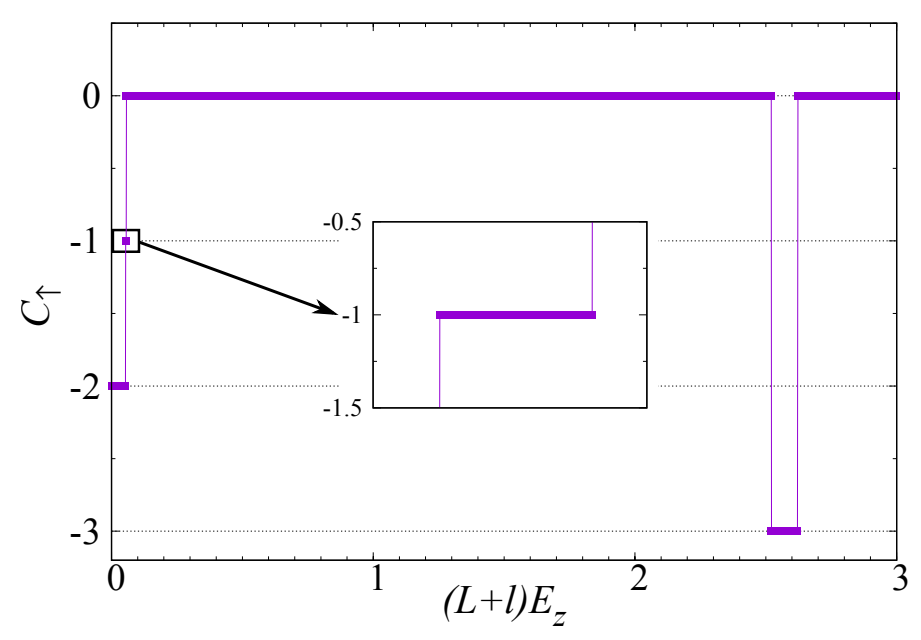

FIG. 4: The Chern number, $C_{\uparrow}$ plotted as a function of $(L+l) E_{z}$ for $\uparrow \operatorname{spin}(\sigma=1)$ in bilayer silicene. Due to the time-reversal symmetry of the model, $C_{\downarrow}=-C_{\uparrow}$.

The values of inter-layer couplings are $t_{\perp}=0.12 t$,

$$
t_{3}=0.1 t \text { and } l=L / 4 \text {. }
$$

The low-energy Hamiltonian expanded around the $K$ and $K^{\prime}$ points in the Brillouin zone then reads, 

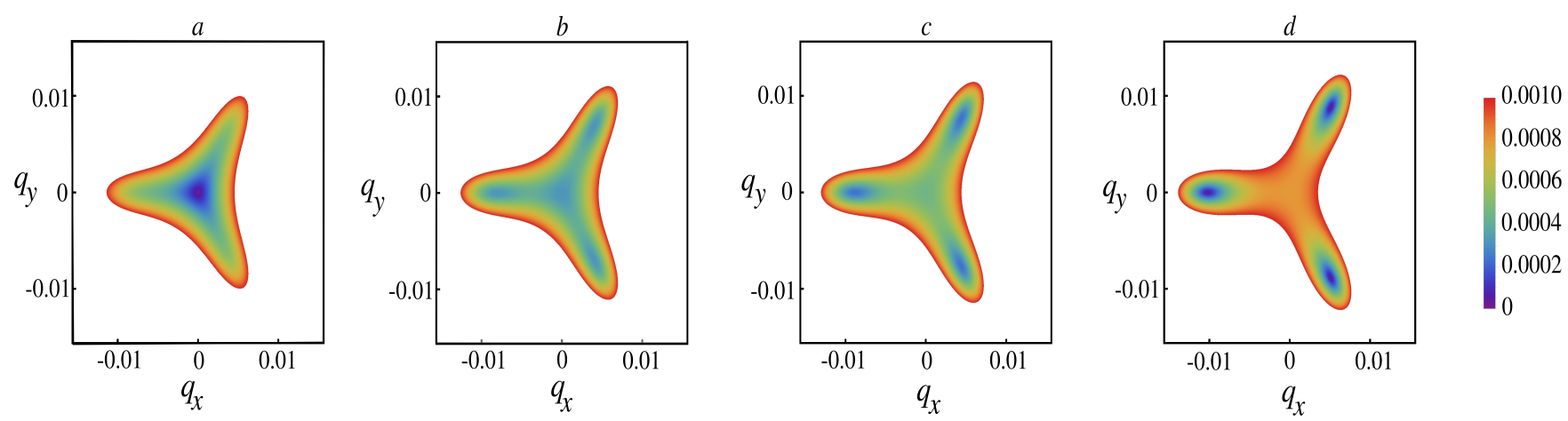

FIG. 5: The plots from left to right corresponds to panels (a)-(d) in Fig 6 and shows the evolution from a single Dirac cone at $K$ point to three Dirac cones on tuning the external electric field. This region is marked by the Chern number -1 in the inset of Fig 4

$$
H_{\eta}=\psi_{q}^{\dagger}\left(\begin{array}{cccc}
\eta \sigma \lambda+(L+l) E_{z} & \frac{3 a t}{2}\left(\eta q_{x}-i q_{y}\right) & 0 & -\frac{3 a t_{3}}{2}\left(\eta q_{x}+i q_{y}\right) \\
\frac{3 a t}{2}\left(\eta q_{x}+i q_{y}\right) & -\eta \sigma \lambda+(L-l) E_{z} & t_{\perp} & 0 \\
0 & t_{\perp} & \eta \sigma \lambda-(L-l) E_{z} & \frac{3 a t}{2}\left(\eta q_{x}-i q_{y}\right) \\
-\frac{3 a t_{3}}{2}\left(\eta q_{x}-i q_{y}\right) & 0 & \frac{3 a t}{2}\left(\eta q_{x}+i q_{y}\right) & -\eta \sigma \lambda-(L+l) E_{z}
\end{array}\right) \psi_{q}
$$

with the same $\psi_{q}, \psi_{q}^{\dagger}$ defined earlier. Note that because of the spin-orbit coupling, this Hamiltonian has an explicit dependence on the spin index $\sigma$. So for each value of $\sigma=\uparrow, \downarrow$, we get a specific $H_{\eta}$. Note also that this reduces to the Hamiltonian in Ref.14 in the appropriate limit.

We can now obtain the eigenvalues of this Hamiltonian as

$$
\begin{aligned}
\epsilon_{q, \eta, \sigma} & = \pm \frac{1}{\sqrt{2}}\left(2\left(L^{2}+l^{2}\right)^{2} E_{z}^{2}+\frac{9 a^{2} q^{2}}{4}\left(2 t^{2}+t_{3}^{2}\right)+t_{\perp}^{2}+2 \lambda^{2}+4 l E_{z} \eta \sigma \lambda\right. \\
& \pm\left(t_{\perp}^{4}+9 a^{2} q^{2} t^{2}\left(4 L^{2} E_{z}^{2}+t_{\perp}^{2}\right)+\frac{9 a^{2} q^{2} t_{3}^{2}}{4}\left(9 a^{2} q^{2}\left(t^{2}+\frac{t_{3}^{2}}{4}\right)-2 t_{\perp}^{2}\right)-27 \eta a^{3} t^{2} t_{3} t_{\perp} q_{x}\left(q_{x}^{2}-3 q_{y}^{2}\right)\right. \\
& \left.\left.+4 L E_{z}\left(\eta \sigma \lambda+l E_{z}\right)\left(\frac{9}{2} a^{2} q^{2} t_{3}^{2}-2 t_{\perp}^{2}\right)+16 L^{2} E_{z}^{2}\left(\eta \sigma \lambda+l E_{z}\right)^{2}\right)^{1 / 2}\right)^{1 / 2}
\end{aligned}
$$

Comparing Eqs. 5 and 11 , we note that they differ by terms proportional to the buckling as well as by the spinorbit coupling terms. Moreover, the eigenvalues are explicitly spin-dependent. At the $K / K^{\prime}$ points, the eigenvalues have the form,

$$
\begin{aligned}
\epsilon_{\eta, \sigma}= & \pm\left(\eta \sigma \lambda+(L+l) E_{z}\right), \\
& \pm \sqrt{\left(\eta \sigma \lambda-(L-l) E_{z}\right)^{2}+t_{\perp}^{2}} .
\end{aligned}
$$

The most important difference between bilayer graphene and silicene is the gap due to the spin-orbit coupling. Like in the case of monolayer silicene, this gap can be closed by tuning the external electric field and leading to Chern number changes which are depicted in Fig 4 .
As the strength of the external electric field increases from zero, the Chern number changes four times corresponding to four gap closings in the Brillouin zone. The first change in the Chern number occurs when the gap closes at $K / K^{\prime}$ points when $(L+l) E_{z}=-\eta \sigma \lambda$, evident from Eq12. The Chern number changes from -2 to -1 here. The second one occurs when the gap closes at the three (expected from trigonal symmetry) satellite Dirac points around $K / K^{\prime}$ points, as $E_{z}$ increased slightly. The Chern number goes from -1 to 0 as shown in the inset of Fig 4 . The evolution of the bands from when the gap closes at the $K$ point (for the $\uparrow$ spin sector) to when the gap closes at the satellite Dirac points as a function of increasing $E_{z}$ is depicted in Fig 5 . This is also the region 

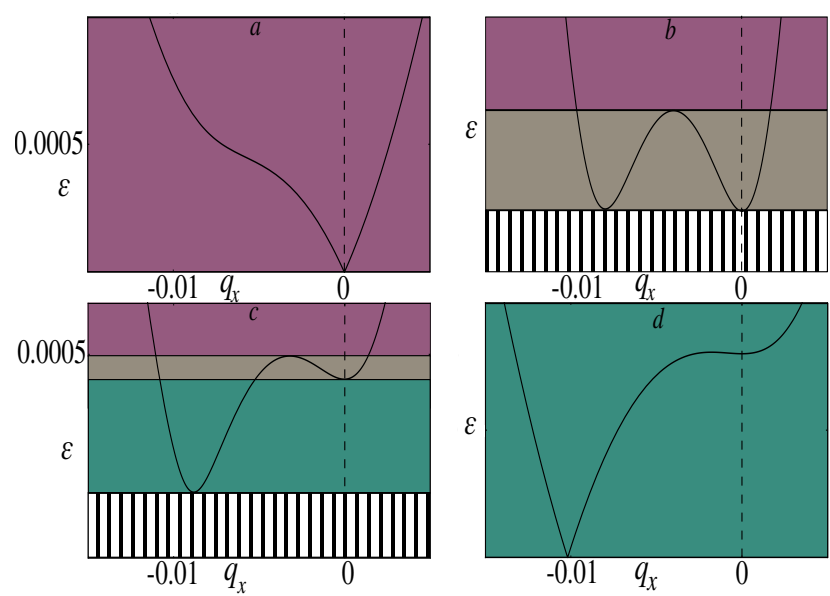

FIG. 6: The Lifshitz phase diagram of bilayer silicene.

The four panels show the evolution of the band structure on the $q_{y}$ axis (for $q_{x}=0$ ) near $K$ as the electric field $E_{z}$ is varied. Panels (a) and (d) have the band crossings at $K$ and satellite Dirac point respectively, while panels (b)-(c) are band structure evolutions between them. The different Lifshitz phases for varying chemical potential values are marked by different colored regions in the phase diagram.

where we choose to study the Lifshitz transition in this model. The third and fourth gap closings happens for much larger values of $E_{z}$ elsewhere in the Brillouin zone which are not accessible in the low energy Hamiltonian in $\mathrm{Eq} 10$.

In Fig.6, we numerically show the evolution of the gaps at the $K$ and at the associated satellite point situated along the $q_{y}=0$ axis. The two other satellite Dirac points are found using the trigonal symmetry. We now try to understand the Lifshitz transition in bilayer silicene and the changes that occur in the Fermi surface topology. We have chosen to show the results for the spin $\uparrow$ sector. The results for the spin $\downarrow$ sector are qualitatively similar.

When the chemical potential is set to zero along with the applied electric field the system has a gap of the $O(\lambda)$. On increasing the strength of the applied electric field, the gap closes at the Dirac point. This gapless band is plotted in Fig 6(a). By increasing the Fermi level from zero, we obtain a Fermi surface with a disc topology or topology of a single region. This Lifshitz phase is marked by purple colored regions in Fig 6(a)-(c). Increasing the electric field further gaps out the Dirac cone at $K$ point (for $\mu=0$ ) while simultaneously creating electron pockets at the three satellite Dirac points. Fig.6(b)-(c) depicts two such cases. Depending on the value of Fermi energy we can get two additional Lifshitz phases along with an insulating phase here. The striped regions in Fig6(b)-(c) are insulating phases where Fermi energy lies below both the electron pockets. The brown regions are the phases with four disconnected Fermi surfaces and the green ones are the phases with three disconnected Fermi surfaces. Further increase of $E_{z}$ results in the gap closing at the satellite Dirac points as shown in Fig.6(d). A non-zero chemical potential here will give a Fermi surface with three disconnected regions.

\section{PERIODIC DRIVING AND THE EFFECTIVE HAMILTONIAN FOR BILAYER MATERIALS}

In this section, we include the effects of periodic driving in the high frequency limit, by obtaining an effective static Hamiltonian using the Brillouin-Wigner (BW) perturbation theory. Generalizing earlier work on single layer systems like graphene ${ }^{\sqrt[35]{5}}$ and silicene $e^{36}$, we find that for the bilayer Hamiltonian described in Eq. 1 , up to $O(1 / \omega)$, the Hamiltonian is given by

$$
H^{\mathrm{B}-\mathrm{W}}=H^{(0)}+H^{(1)}
$$

where $H^{(0)}$ and $H^{(1)}$ are the zeroth and first order terms in the B-W expansion. The effect of radiation is taken into account by the vector potential $\mathbf{A}(\tau)=$ $A_{0}(\cos \omega \tau, \sin \omega \tau)$, where $\omega$ is the frequency and we work in the high frequency limit. The hopping term is then modified by the Peierls substitution and changes to $-t \sum_{\langle i, j\rangle} e^{-i \alpha \sin (\omega \tau-2 \pi l / 3)} a_{i}^{\dagger} b_{j}$, where $l=0,1,2$ for the three nearest neighbours in the honeycomb lattice. We will generically include both buckling and the spin-orbit terms and then set them to zero when considering bilayer graphene and use the appropriate values for the different spin-orbit coupled materials. The expansion has terms re normalizing the intra-layer couplings of both the layers and the inter-layer terms between them. The renormalization of the in-plane terms are given by 


$$
\begin{aligned}
& \mathcal{J}_{\sigma}=-t J_{0}(\alpha)+\frac{4 t \sigma \lambda}{3 \omega} \sum_{n \neq 0} \beta_{n} \sin \frac{\pi n}{6}+\frac{t^{3}}{\omega^{2}}\left[\sum_{n \neq 0} \gamma_{n}\left(2 \cos \frac{2 \pi n}{3}+3\right)+\sum_{m, n \neq 0} \chi_{n m}\left(4 \cos \frac{2 \pi n}{3}+1\right)\right] \\
& \Lambda_{\sigma}^{0}=\frac{\sigma \lambda J_{0}(\alpha \sqrt{3})}{3 \sqrt{3}}-\sum_{n \neq 0} \frac{t^{2} J_{n}^{2}(\alpha)}{\omega n} \sin \frac{2 \pi n}{3} \\
& L_{\sigma}=-\frac{4 t \sigma \lambda}{3 \omega} \sum_{n \neq 0} \beta_{n} \sin \frac{\pi n}{2}+\frac{2 t^{3}}{\omega^{2}}\left(\sum_{n \neq 0} \gamma_{n} \cos \frac{2 \pi n}{3}+\sum_{m, n \neq 0} \chi_{n m} \cos \frac{2 \pi(m-n)}{3}\right) \\
& M_{\sigma}=-\frac{2 t \sigma \lambda}{3 \omega} \sum_{n \neq 0} \beta_{n} \cos \pi n \sin \frac{\pi n}{6}+\frac{t^{3}}{\omega^{2}}\left(\sum_{n \neq 0} \gamma_{n} \cos \frac{2 \pi n}{3}+\sum_{m, n \neq 0} \chi_{n m} \cos \frac{2 \pi(m+n)}{3}\right)
\end{aligned}
$$

where the original parameters $t$ and $\lambda$ are defined in Eqs. 2 and 8 and $\alpha=a A . J_{n}$ is the Bessel function of order $n$ and $\beta_{n}=J_{n}(\alpha) J_{n}(\alpha \sqrt{3}) / \sqrt{3} n, \gamma_{n}=J_{n}^{2}(\alpha) J_{0}(\alpha) / n^{2}$ and $\chi_{n m}=J_{m}(\alpha) J_{n}(\alpha) J_{m+n}(\alpha) / m n$.

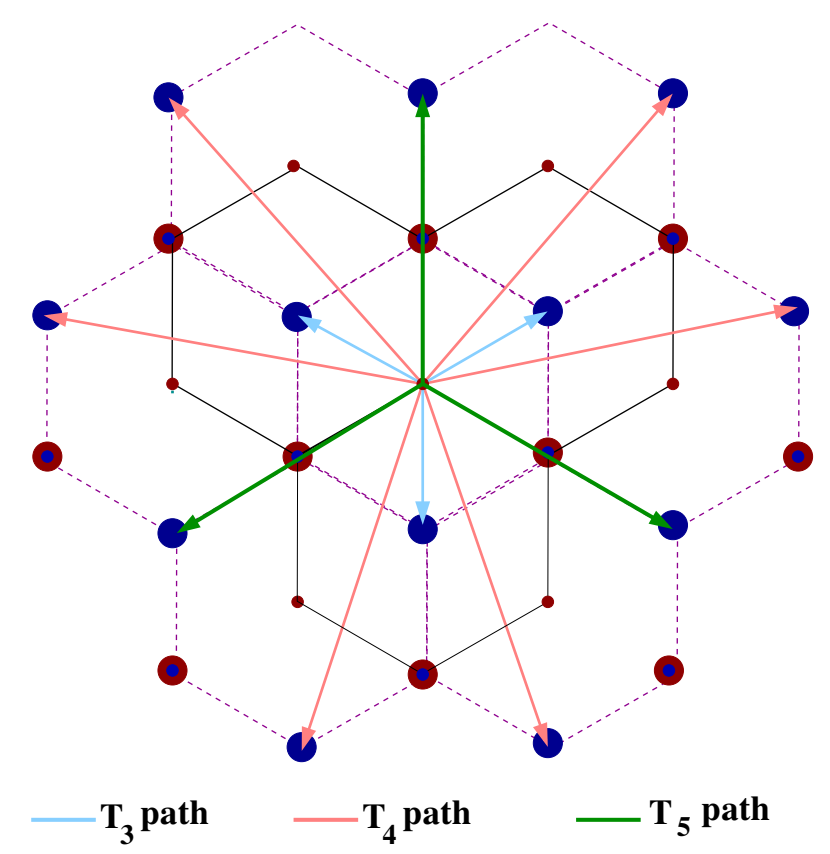

FIG. 7: Different hopping paths between layers in bilayer silicene

The inter-layer Hamiltonian $H_{\text {inter }}$ can also be expanded order by order, leading to longer ranged hoppings as shown in Fig.7. The zeroth order term is given by

$$
\begin{aligned}
H^{(0)} & =t_{\perp} \sum_{i \in \tilde{A}, j \in B}\left(\tilde{a}_{i \sigma}^{\dagger} b_{j \sigma}+b_{j \sigma}^{\dagger} \tilde{a}_{i \sigma}\right) \\
& +t_{3} J_{0}(\alpha)\left(\sum_{i \in A} a_{i \sigma}^{\dagger} \tilde{b}_{i-\delta_{l} \sigma}+\sum_{j \in \tilde{B}} \tilde{b}_{j \sigma}^{\dagger} a_{j+\delta_{l} \sigma}\right) .
\end{aligned}
$$

The $t_{\perp}$ term exists only in the zeroth order term and does not occur at $O(1 / \omega)$.

The first order term, $O(1 / \omega)$ involving $t_{3}$ is given by,

$$
\begin{aligned}
H_{B W}^{(1)}= & \sum_{\left\{n_{i}\right\} \neq 0} \frac{H_{0, n_{1}} H_{n_{1}, 0}}{n_{1} \omega} \\
= & \sum_{\{n\} \neq 0} \frac{1}{n \omega}\left[T_{-n}^{3} T_{n}^{3}+T_{-n} T_{n}^{3}+\tilde{T}_{-n} T_{n}^{3}+T_{-n}^{3} \tilde{T}_{n}\right. \\
& \left.+T_{-n}^{3} T_{n}\right] .
\end{aligned}
$$

Here, the terms of $O\left(t t_{3}\right)$ cancel and the terms of $O\left(t_{3}^{2}\right)$ renormalizes the spin-orbit coupling terms in the $A$ sublattice of the top layer and the $\tilde{B}$ sublattice of the bottom layer as follows -

$$
\Lambda^{1}=-i \frac{t_{3}^{2} J_{n}^{2}(\alpha)}{n \omega} \sin \frac{2 n \pi}{3} \sum_{\langle\langle i, j\rangle\rangle} \nu_{i j}\left(a_{i}^{\dagger} a_{j}+\tilde{b}_{i}^{\dagger} \tilde{b}_{j}\right) .
$$

The terms $O\left(\lambda t_{3}\right)$ contribute the following three terms to the effective $H$ -

$$
\begin{aligned}
& T_{3}^{\prime}=\frac{4 t_{3} \lambda \sigma}{3 \sqrt{3} \omega n} J_{n}(\alpha \sqrt{3}) J_{n}(\alpha) \sin \frac{n \pi}{6} \sum_{\substack{i \in A j \in \tilde{B} \\
\langle\langle\langle i, j\rangle\rangle\rangle}}^{T_{3}-\text { path }} a_{i}^{\dagger} \tilde{b}_{j} \\
& T_{4}=\frac{-2 t_{3} \lambda \sigma}{3 \sqrt{3} \omega n} J_{n}(\alpha \sqrt{3}) J_{n}(\alpha) \sin \frac{n \pi}{6} \cos n \pi \sum_{\substack{i \in A j \in \tilde{B} \\
\langle\langle i, j\rangle\rangle\rangle}}^{T_{4}-\text { path }} a_{i}^{\dagger} \tilde{b}_{j} \\
& T_{5}=\frac{-4 t_{3} \lambda \sigma}{3 \sqrt{3} \omega n} J_{n}(\alpha \sqrt{3}) J_{n}(\alpha) \sin \frac{n \pi}{2} \sum_{\substack{i \in A j \in \tilde{B} \\
\langle\langle\langle i, j\rangle\rangle\rangle}}^{T_{5}-\text { path }} a_{i}^{\dagger} \tilde{b}_{j}
\end{aligned}
$$

The $T_{3}^{\prime}$ renormalizes the $t_{3}$ term in the bare Hamiltonian, whereas the $T_{4}$ and $T_{5}$ couplings are longer ranged interlayer hopping terms depicted in Fig.7.

The effective Hamiltonian for bilayer materials in momentum space with renormalized interactions is given by 


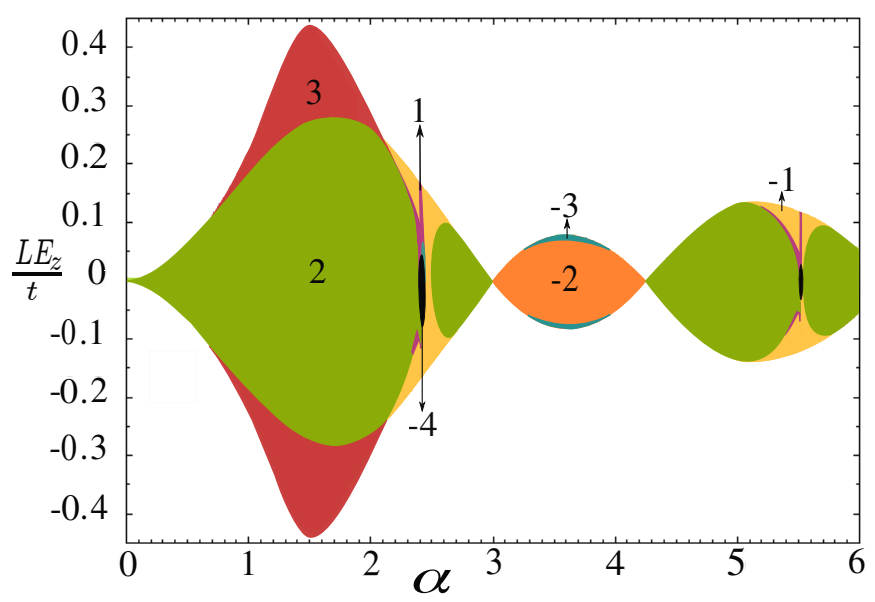

FIG. 8: The Floquet topological phase diagram of bilayer graphene as a function of the drive amplitude $\alpha$.

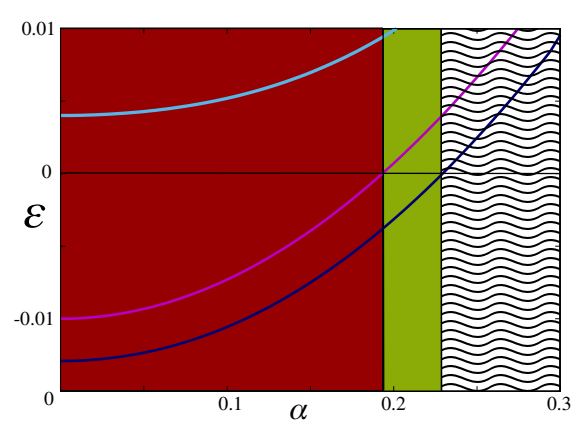

(a) $L E_{z}=0.01 t, \mu=0.02 t$

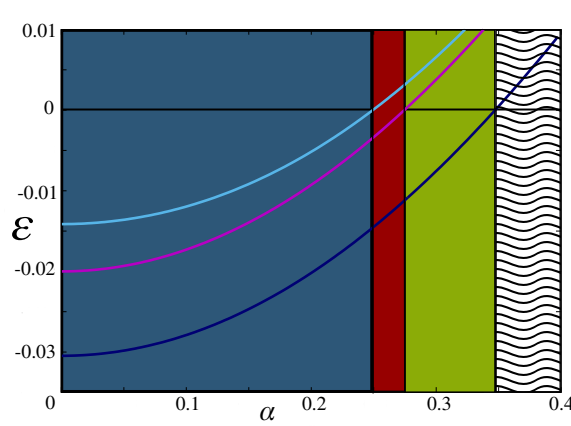

(b) $L E_{z}=0.03 t, \mu=0.05 t$

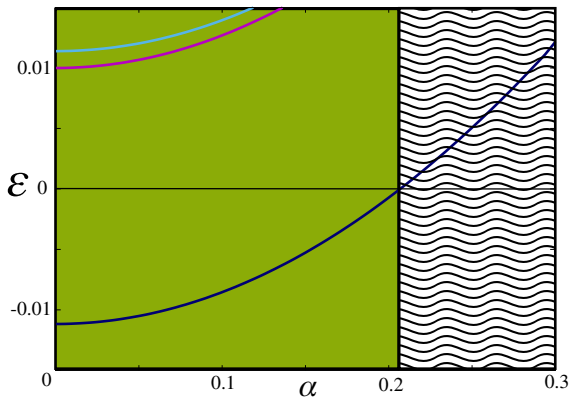

(c) $L E_{z}=0.06 t, \mu=0.05 t$

FIG. 9: The Floquet Lifshitz phase diagram of bilayer graphene. The change in phases with increasing $\alpha$ is plotted for different initial values of $L E_{z}$ and $\mu$. The colors of the different phases are the same as that in Fig 2

$$
H_{\mathrm{eff}}=\left(\begin{array}{cccc}
\Lambda_{q \sigma}^{0}+\Lambda_{q \sigma}^{a}+\Lambda_{q \sigma}^{1}+\mu_{q \sigma}^{a} & \mathcal{J}_{q \sigma}+L_{q \sigma}+M_{q \sigma} & 0 & \tilde{T}_{3 q \sigma} \\
\mathcal{J}_{q \sigma}^{*}+L_{q \sigma}^{*}+M_{q \sigma}^{*} & -\Lambda_{q \sigma}^{0}+\Lambda_{q \sigma}^{b}+\mu_{q \sigma}^{b} & t_{\perp} & 0 \\
0 & t_{\perp} & \Lambda_{q \sigma}^{0}+\Lambda_{q \sigma}^{\tilde{a}}+\mu_{q \sigma}^{\tilde{a}} & \mathcal{J}_{q \sigma}+L_{q \sigma}+M_{q \sigma} \\
\tilde{T}_{3 q \sigma}^{*} & 0 & \mathcal{J}_{q \sigma}^{*}+L_{q \sigma}^{*}+M_{q \sigma}^{*} & -\Lambda_{q \sigma}^{0}-\Lambda_{q \sigma}^{1}+\Lambda_{q \sigma}^{\tilde{b}}+\mu_{q \sigma}^{\tilde{b}}
\end{array}\right)
$$

where $T_{3}+T_{3}^{\prime}+T_{4}+T_{5}=\tilde{T}_{3 q \sigma}$ and

$$
\begin{aligned}
\Lambda_{\sigma}^{a / b} & =-\frac{t^{2}\left((L \pm l) E_{z}-\mu\right)}{\omega^{2}}\left(\sum_{n \neq 0} \frac{J_{n}^{2}(\alpha)}{n^{2}} \cos \frac{2 \pi n}{3}\right) \\
\Lambda_{\sigma}^{\tilde{a} / \tilde{b}} & =-\frac{t^{2}\left((-L \pm l) E_{z}-\mu\right)}{\omega^{2}}\left(\sum_{n \neq 0} \frac{J_{n}^{2}(\alpha)}{n^{2}} \cos \frac{2 \pi n}{3}\right) \\
\mu_{\sigma}^{a / b} & =\left(1-\frac{3 t^{2}}{\omega^{2}} \sum_{n \neq 0} \frac{J_{n}^{2}(\alpha)}{n^{2}}\right)\left((L \pm l) E_{z}-\mu\right) \\
\mu_{\sigma}^{\tilde{a} / \tilde{b}} & =\left(1-\frac{3 t^{2}}{\omega^{2}} \sum_{n \neq 0} \frac{J_{n}^{2}(\alpha)}{n^{2}}\right)\left((-L \pm l) E_{z}-\mu\right)
\end{aligned}
$$

Using this effective Hamiltonian, we will now study the effect of driving at high frequency in both bilayer graphene and bilayer silicene and obtain its effects in the next section.

\section{THE PHASE DIAGRAMS}

In this section, we show how high frequency light can be used to obtain new phases and control changes in the Fermi surface topology, in both bilayer graphene and bi- 
layer silicene. For single layer materials, we already know that shining light leads to changes in the Chern number and hence leads to several new phases. Here, we wish to study changes in Chern number as well as changes in Fermi surface topology as a function of the amplitude of light.

\section{A. Bilayer graphene}

The Hamiltonian in Eq. (19) has terms involving spinorbit coupling and a buckled lattice structure. These are set to zero for bilayer graphene, i.e., we set $\tilde{T}_{3 q \sigma}=T_{3}$, since there is neither buckling nor any spin dependence, and $l=0$, since there is no spin-orbit coupling, along with $\mu=0$ in Eq20. This means that $\Lambda^{a}=\Lambda^{b}$ and $\Lambda^{\tilde{a}}=\Lambda^{\tilde{b}}$ with $\Lambda^{a}=-\Lambda^{\tilde{a}}$. Similarly $\mu^{a}=\mu^{b}$ and $\mu^{\tilde{a}}=\mu^{\tilde{b}}$ with $\mu^{a}=-\mu^{\tilde{a}}$. The values of the inter-layer couplings are taken as $t_{\perp}=0.12 t$ and $t_{3}=0.1 t$.

We then expand the Hamiltonian around the $K$ and $K^{\prime}$ points and calculate the energy eigenvalues to study whether the trigonal warping is modified in the effective Hamiltonian. For simplicity, we set $L E_{z}=0$ in the original undriven Hamiltonian. The position of the four gapless Dirac cones then reduces to that given in Eq. 6. with $L E_{z}=0$ which simplifies the effective B-W Hamiltonian in Eq. 19. The only new remaining terms in the diagonal as compared to the static case, $\Lambda^{0}, \Lambda^{1}$ are 'effective spinorbit couplings' and are, therefore, momentum independent in the low energy limit. Thus, even in the presence of driving, the functional form of the eigenvalues in Eq 5 does not change. However, the 'effective spin-orbit coupling terms' introduced by driving leads to a mass gap, given by $2\left|\Lambda^{0}+\Lambda^{1}\right|$ just like it does for genuinely spinorbit coupled materials like silicene. Moreover, since this gap is introduced by the driving, it is dependent on the amplitude $\alpha$ of the light and is oscillating. These oscillations lead to gap closures and Chern number changes as shown in Fig 8 .

We then compute the Chern numbers for a range of values of $E_{z}$ and $\alpha$ for $\omega=10 t$ to construct a phase diagram describing the various topological phases in this model shown in Fig, 8 . The phase diagram is symmetric under $L E_{z} \rightarrow-L E_{z}$ in most parts of the phase diagram other than the regions near $\alpha=2.4$ and 5.5, although the terms $\Lambda^{a}$ and $\mu^{a}$ in the Hamiltonian change their sign under this symmetry. However, since both these terms are very small compared to the other terms, this symmetry breaking is only visible when the all other terms are also small. Other than that, the phase diagram shows the expected repetition of phases (with smaller areas) as we increase the value of the amplitude $\alpha$ of light $t^{36}$.

To study the Lifshitz transition using the B-W effective Hamiltonian, we consider three different initial points in the Lifshitz phase diagram of bilayer graphene in Fig 2 and study the effect of shining light on these phases. The inter-layer coupling values are taken as $t_{\perp}=0.5 t$, $t_{3}=0.45 t$ which are same as in Fig 2. The resultant phase diagrams as a function of the amplitude of light are depicted in Fig.9. The (a) magenta, (b) dark blue and (c) light blue curves are the heights (measured from $\varepsilon_{F}=0$ to the bottom of the cone) of (a) the Dirac cone at the $K$ point, (b) the satellite Dirac cone and (c) the van-Hove singularity, respectively. The colours in this phase diagram are chosen to match the ones in the phase diagram without light Fig2, In Fig 9a, in the red region, both the Dirac point and the satellite Dirac points are below the Fermi level. The topology is that of four disconnected regions in the Fermi level. As the value of $\alpha$ increase from zero, close to $\alpha=0.2$, the Dirac cone rises above the Fermi level changing the topology to that of three disconnected regions. This is the green region in this phase diagram and in Fig2, As $\alpha$ is further increased, the satellite cones arises above the Fermi level and we enter the insulating striped phase. Thus, as a function of driving, we are able to tune a Lifshitz (topology-changing) transition.

In Figs. 9b and 9c we start from initial points in the blue and green phases in Fig 2 and show the changes that occur under driving as shown in Fig9. In Fig 9b, we start from the phase where even the van-Hove singularities is below the Fermi level. By shining light, we first move to a phase with 4 disconnected regions (red phase) where the van-Hove singularities go above the Fermi level. Further driving moves the Dirac point above the Fermi level and we reach the green phase with three disconnected regions and finally, when even the satellite Dirac points go above the Fermi level, we reach the insulating phase. In Fig 9c we start from the green phase where only the three satellite Dirac points are below the Fermi level and directly transition into the insulating phase.

\section{B. Bilayer spin-orbit coupled materials}

In this section, we study the Floquet phase diagram and the Lifshitz transition for spin-orbit coupled materials. The Floquet topological phase diagram for $\uparrow$ spin is calculated in Fig 10 for $\lambda=0.05 t, \omega=10 t, t_{\perp}=0.12 t$, $t_{3}=0.1 t$ and $L=4 l$ as a function of $E_{z}$ and $\alpha$. The phase diagram can be divided into three regions with topological phases separated by trivial ones as we increase the perpendicular electric field. The top and bottom regions corresponds to the $C_{\uparrow}=-3$ phase in the static phase diagram of silicene in Fig 4 and exists for both positive and negative values of $E_{z}$. This phase continues to have a non-zero Chern number $(3,-3)$ until $\alpha$ is increased to $\approx 1.25$ before disappearing as shown in the inset. The middle region is similar to that of bilayer graphene in Fig, 8 and to that of the topological phase diagram of irradiated single layer silicene studied using B-W theory ${ }^{36}$. The $C_{\uparrow}=-2$ phase from Fig 4 extends for a small region in $\alpha$ while $C_{\uparrow}=-1$ region is too narrow to be visible in a large phase diagram.

In the rest of this section, we try to understand the effect of an additional tuning parameter $\alpha$ on the Lifshitz 


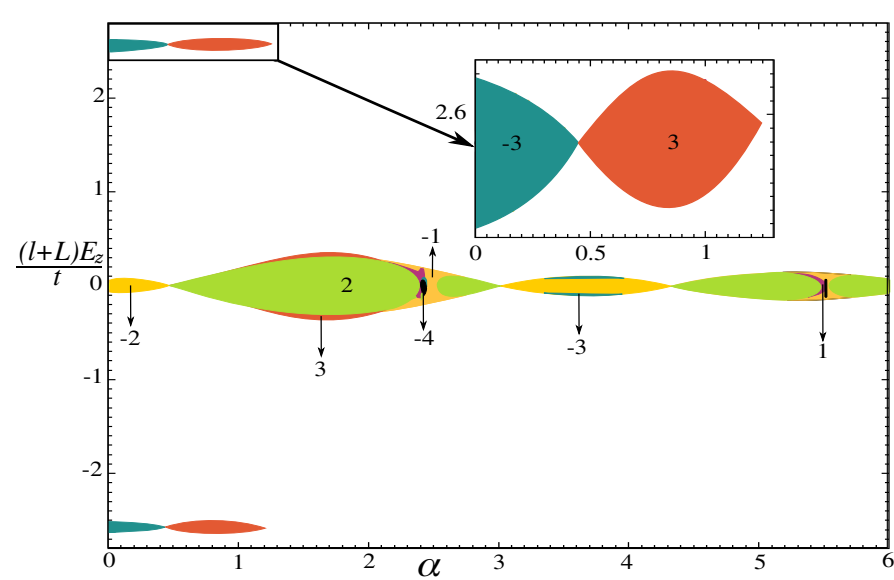

FIG. 10: Floquet topological phase diagram of bilayer materials with spin-orbit coupling. The phase diagram is plotted for up spin.
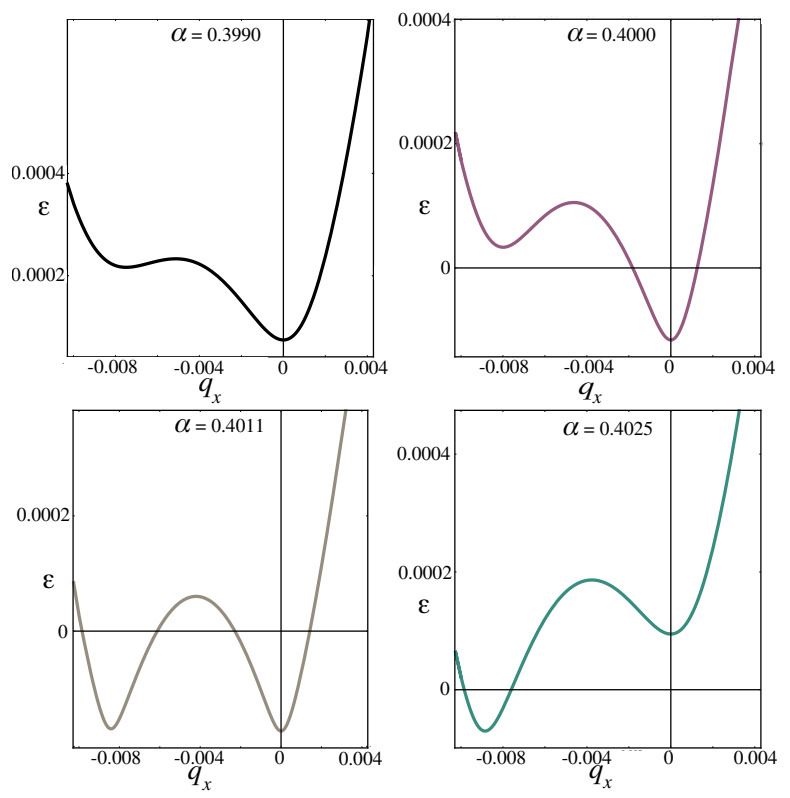

FIG. 11: Floquet Lifshitz phase diagram for bilayer silicene with varying $\alpha$ for $(L+l) E_{z}=-0.01 t$ and

$$
\mu=0.00025 t \text {. }
$$

phase transition of bilayer silicene. To compare it with the bare case described in Fig 6 , we fix the values of $\mu$ and $E_{z}$ and tune the value of $\alpha$ as shown in Fig.11. For $\mu / t=$ 0.00025 and $(L+l) E_{z} / t=-0.01$, we have considered four values of $\alpha$ in Fig 11 . In the four different panels, the color of the curve is same as the color of the Lifshitz phase in Fig 6 for that particular value of $\alpha$. The top left panel where $\alpha=0.3990$ corresponds to the striped phase in Fig6. This is an insulating phase where the Fermi energy is in the gap. The top right panel with $\alpha=0.4000$ and the purple curve corresponds to the phase with the same color in Fig.6. Here the electron pocket at the Dirac $K\left(K^{\prime}\right)$ point is below the Fermi level and the topology is that of a disc corresponding to a single region in the Fermi surface. The bottom left and the bottom right panels with $\alpha=0.4011$ and $\alpha=0.4025$ correspond to the brown and green colored phases respectively in Fig, 6 . The brown colored phase has the electron pockets at the Dirac point and at the three satellite points below the Fermi level. This implies that the the Fermi surface is made up of four disconnected regions. The green colored region consists of three disconnected regions since the electron pocket at the satellite points are below the Fermi level. Hence, as a function of the drive, we can tune the bilayer silicene through topology changing Lifshitz transitions - from an insulating phase in (a) to a single region Fermi surface in (b) to a four region Fermi surface in (c) and finally to a three region Fermi surface in (d). Note also that these transitions are extremely sensitive to the value of the amplitude of the drive and occur for very small changes in $\alpha$. This is not too surprising because these changes are correlated with changes in the Chern number at the same value of $\alpha$ in Fig.10, and in that figure, the region of the phase change from -2 to -1 to 0 is so small that it cannot be shown in the figure, where it looks like the phase change is directly from -2 to 0 .

\section{DISCUSSION AND CONCLUSIONS}

In this paper, we have studied the effect of light on bilayer graphene and silicene and seen how they affect the Fermi-surface topology changing Lifshitz transitions. Physically, it is the magnetotransport properties, such as the Shubnikov-de Haas effect and thermodynamic properties such as the de Haas-van Alphen effect which are affected by the changes in Fermi surface topology. Essentially, when there are either creation of additional Fermi surface pockets or merging of Fermi surface pockets, the Landau level degeneracy changes. For instance, when the Fermi level decreases from the blue region in Fig 2 with a single Fermi surface to the green region, with three Fermi surface pockets, the period of the oscillations triple. So changes in the degeneracy which are caused either by the Lifshitz transitions can be easily measured by the Shubnikov-de Haas oscillations period. Moreover, precisely at the multicritical point (black dot) in Fig 2 where the critical points merge, the density of states diverges. This is also something which can be seen experimentally. Finally, in this paper, we have also shown how these phases in bilayer graphene and in spin-orbit coupled materials like bilayer silicene, can be controlled by shining light on these systems, paving the way for optoelectronic devices in these materials.

During the preparation of this paper for publication, we became aware of the work of Ref 43 who also study the effect of light on Lifshitz transition in bilayer graphene. Our results agree where there is overlap. 


\section{ACKNOWLEDGMENTS}

We would like to thank Abhishek Joshi, Arijit Kundu and Ganpathy Murthy for useful discussions. PM would like to thank HRI for hospitality during the completion of this work.
${ }^{1}$ K. S. Novoselov et al, Nature 438, 197 (2005).

2 K. S. Novoselov et al, Nature Phys. 2, 177 (2006).

3 E. McCann and V. I. Falko, Phys. Rev. Lett.96, 086805 (2006).

4 F. Guinea, A. H. Castro Bero and N. M. R. Peres, Phys. Rev. B73 245426 (2006).

5 S. Latil and L. Henrard, Phys. Rev. Lett.97, 036803 (2006).

6 B. Partoens and F. M. Peeters, Phys. Rev. B74, 075404 (2006).

7 B. Partoens and F. M. Peeters, Phys. Rev. B75, 193402 (2007).

8 For more recent theoretical studies of bilayer graphene, see M. Koshino, New Jnl of Physics, 11, 095010 (2009) and references therein.

9 M. Ezawa, Phys. Rev. Lett.110, 026603 (2013).

10 N. D. Drummond, V. Zolyomi and V. I. Falko, Phys. Rev. B85, 075423 (2012).

11 C. C. Liu, H. Jiang and Y. Yao, Phys. Rev. B84, 195430 (2011).

12 C. C. Liu, W. Feng and Y. Yao, Phys. Rev. Lett.107, 076802 (2011).

13 M. Ezawa, J. Phys. Soc, Jpn, 84, 121003 (2015).

14 M. Ezawa, Jnl of Phys. Soc. Jpn, 81, 104713 (2012).

15 H. Fu, J. Zhang, Z. Ding, H. Li and S. Meng, App. Phys. Lett. 104, 131904 (2014).

16 J. E. Padilha and R. B. Pontes, J. Phys. Chem. C119, 3318 (2015).

17 M. Zhang, L. Xu and J. Zhang, J. of Phys : Cond. Matt. 27, 445301 (2015).

18 T. Do, P. Hsih, G. Gumbs, D. Huang, C. Chiu and M. Lin, Phys. Rev. B97, 125416 (2018)

19 N. Goldman and J. Dalibard, Phys. Rev. X, 031027 (2014).

20 M. Bukov, L. DAlessio, and A. Polkovnikov, Advances in Physics 64, 139 (2015).

21 M. C. Rechtsman, J. M. Zeuner, Y. Plotnik, Y. Lumer, D. Podolsky, F. Dreisow, S. Nolte, M. Segev, and A. Szameit, Nature 496, 196 (2013).

22 G. Jotzu, M. Messer, R. Desbuquois, M. Lebrat, T. Uehlinger, and D. Greif Nature 515, 237240 (2014)

${ }^{23}$ Y. Wang, H. Steinberg, P. Jarillo-Herrero, and N. Gedik, Science 342, 453 (2013).
${ }^{24}$ T. Oka and H. Aoki, Phys. Rev. B 79, 081406(R) (2009).

25 T. Kitagawa, T. Oka, A. Brataas, L. Fu, and E. Demler, Phys. Rev. B 84, 235108 (2011).

${ }^{26}$ N. H. Lindner, G. Refael, and V. Galitski, Nature Phys. 7, 490 (2011).

27 B. Dora, J. Cayssol, F. Simon, and R. Moessner, Phys. Rev. Lett. 108, 056602 (2012).

28 M. S. Rudner, N. H. Lindner, E. Berg, and M. Levin, Phys. Rev. X 3, 031005 (2013).

29 A. Kundu, H.A. Fertig, and B. Seradjeh, Phys. Rev. Lett. 113, 236803 (2014).

30 G. Usaj, P. M. Perez-Piskunow, L. E. F. Foa Torres, and C. A. Balseiro, Phys. Rev. B 90, 115423 (2014).

31 P. Titum, N. H. Lindner, M. C. Rechtsman, and G. Refael, Phys. Rev. Lett. 114, 056801 (2015).

32 A. Farrell and T. Pereg-Barnea, Phys. Rev. Lett. 115, 106403 (2015).

33 A. Kundu, H.A. Fertig and B. Seradjeh, Phys. Rev. Lett. 116, 016802 (2016).

34 P. Titum, E. Berg, M. S. Rudner, G. Refael, N. H. Lindner, Phys. Rev. X 6, 021013 (2016).

35 T. Mikami, S. Kitamura, K. Yasuda, N. Tsuji, T. Oka, and H. Aoki, Phys. Rev. B 93, 144307 (2016).

${ }^{36}$ P. Mohan, R. Saxena, A. Kundu and S. Rao, Phys. Rev. B94, 235419 (2016).

37 J. Klinovaja, P. Stano, and D. Loss, Phys. Rev. Lett. 116, 176401 (2016).

38 A. Varlet et al, Synthetic Metals, Reviews of current advances in graphene science and technology, Vol. 210, 19 (2015).

39 A. Shtyk, G. Goldstein and C. Chamon, Phys. Rev. B95, 035137 (2017).

40 E. Suarez Morell and L. E. F. Torres, Phys. Rev. B86, 125449 (2012).

41 V. Dal Lago, E. Suarez Morell and L. E. F. Torres, Phys. Rev. B96, 235409 (2017).

42 Y. Yamaji, T. Misawa and M.Imada, Jnl of Phs. Soc. Jpn, 75, 094719 (2006).

${ }^{43}$ I. V. Iorsh, K. Dini, O. V. Kibis and I. A. Shelykh, Phys. Rev. B96, 155432 (2017). 